> L'étonnante diversité des formes biologiques a toujours fasciné à la fois les biologistes et les physiciens. Dès les années 1970, Malcom Steinberg attribuait l'émergence spontanée de formes spécifiques à des différences d'adhérence entre cellules. Cependant, le lien entre les propriétés cellulaires des molécules d'adhérence, telles que les cadhérines, et les lois générales de la physique est resté longtemps flou. Ce n'est que très récemment que cette hypothèse a été validée à la fois par des études in vitro et, de manière spectaculaire, in vivo chez la drosophile. En effet, les outils génétiques puissants disponibles chez la drosophile ont permis de montrer que les lois de la thermodynamique et une régulation génétique simple de l'expression des cadhérines contribuaient à expliquer des processus aussi complexes que l'établissement de l'axe antéro-postérieur ou la morphogenèse de l'œil composé. <

\section{Régulation \\ génétique \\ de l'adhérence \\ intercellulaire \\ Ou comment les cadhérines \\ sculptent la drosophile}

Isabelle Bécam, Jean-René Huynh

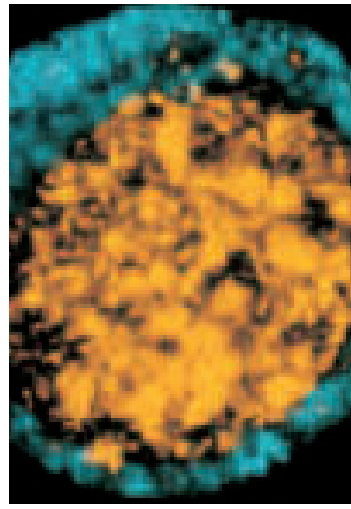

I. Bécam : Institut

Jacques-Monod, CNRS,

Universités Paris 6 et 7 ,

2, place Jussieu,

75251 Paris Cedex 05, France.

Adresse actuelle : ICREA

et Institut de Recerca

Biomedica, Parc Cientific

de Barcelona, Josep Samitier,

1-5, 08028 Barcelona, Espagne.

J.R. Huynh : Institut

Jacques-Monod, CNRS,

fique lui permettant de Universités Paris 6 et 7 , reconnaître de manière sélective les cellules

2 , place Jussieu,

75251 Paris. Cedex 05, France.

huynh@ijm.jussieu.fr

d'un même tissu, et

proposa que l'ordon-

nancement des tissus

au sein de l'embryon provient d'une «affinité tissulaire » différentielle entre les tissus [2]. Ces affinités ont ensuite été associées aux propriétés d'adhérence de la membrane des cellules. Cependant, la nature de cette adhérence et des processus qui guident le mouvement des tissus au cours de la morphogenèse est restée longtemps inconnue [3].

\section{Bases moléculaires et physiques de l'adhérence et de la morphogenèse}

Bases moléculaires de l'adhérence intercellulaire: la famille des cadhérines

II fallut attendre 1977 et l'identification de la première molécule d'adhérence, appelée N-CAM (neuronal-cell adhesion molecule), pour démontrer que l'adhérence cellulaire était due à des protéines spécialisées, localisées à la membrane des cellules [4-6]. Depuis, on distingue l'adhérence entre cellules d'un même tissu, principalement assurée par des protéines appartenant à la super-famille des cadhérines, et l'adhérence des 
cellules à la matrice extracellulaire, qui permet la cohésion des tissus, sous la dépendance des intégrines (pour des revues détaillées, voir [7-9]). Les cadhérines possèdent un large domaine extracellulaire, qui, en présence d'ions calcium, se lie directement au domaine extracellulaire d'une autre molécule de cadhérine de la cellule adjacente (Figure 1). La partie intracytoplasmique des cadhérines classiques est très conservée et contient un site de liaison à la $\beta$-caténine. La $\beta$-caténine, en se liant à l' $\alpha$-caténine, relie les cadhérines directe-

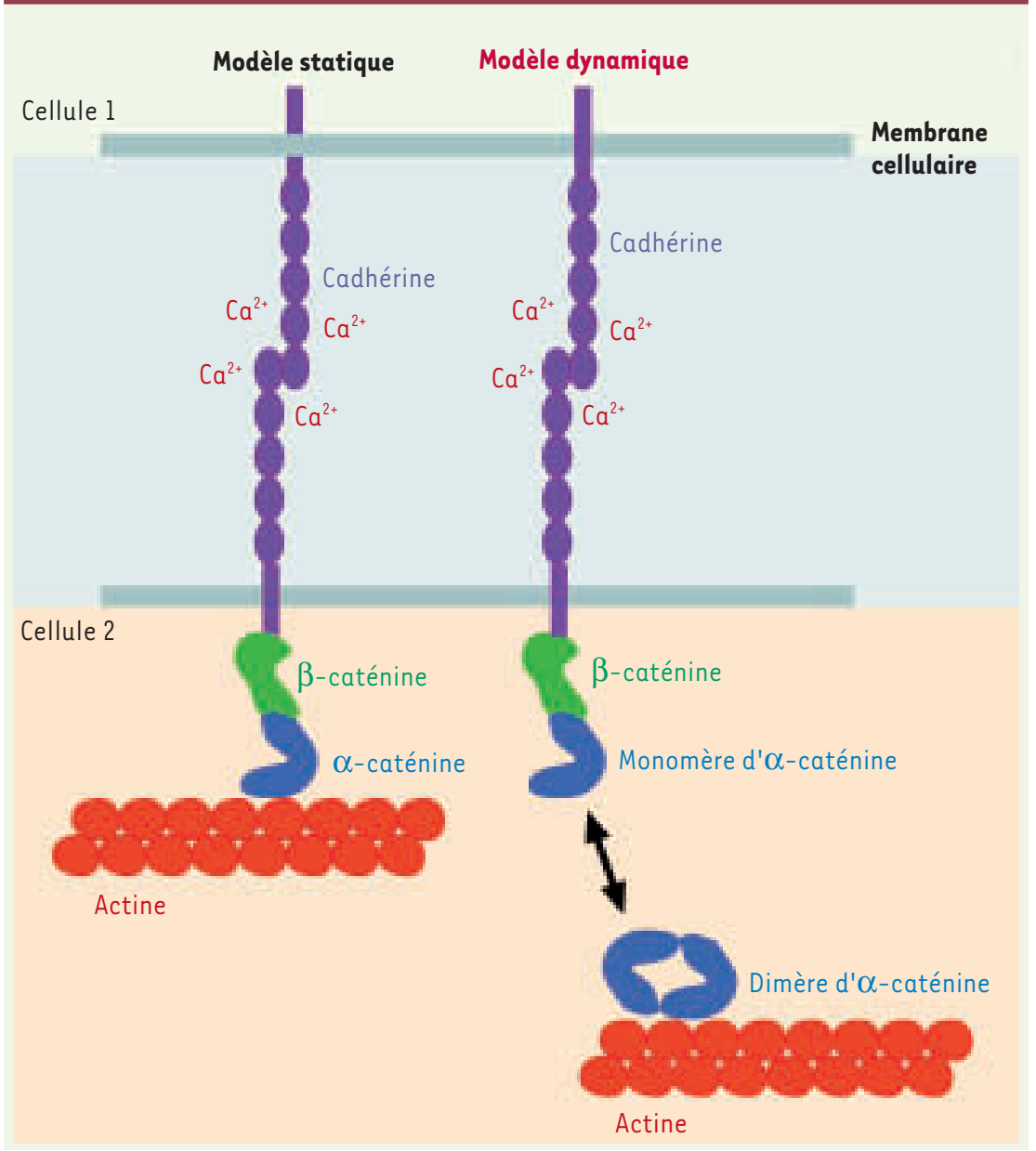

Figure 1. Les cadhérines classiques. Tous les membres de la superfamille des cadhérines sont des protéines transmembranaires qui sont caractérisées par un domaine unique, appelé le domaine cadhérine, qui permet les interactions homophiliques, dépendantes du calcium. Le domaine intracellulaire est lui au contraire très divergent entre les différentes familles mais très conservé au sein d'une même classe. Ainsi, les cadhérines classiques ont un domaine cytoplasmique très conservé. La queue intracytoplasmique de la $\varepsilon$-cadhérine (en violet) se lie à la $\beta$-caténine (vert), ou Armadillo chez la drosophile. La $\beta$-caténine se lie à l' $\alpha$-caténine (bleu) qui elle interagit avec l'actine (rouge). Ainsi, le modèle proposé jusqu'ici était que la $\varepsilon$-cadhérine est reliée à l'actine via les caténines (modèle statique). Cependant, récemment, ce modèle statique a été remis en question [32-34]. En effet, les équipes de Nelson et Weiss ont montré que l' $\alpha$-caténine était associée de manière exclusive soit au complexe $\beta$-caténine/ $\varepsilon$-cadhérine soit à l'actine (modèle dynamique). Les auteurs proposent ainsi un modèle plus dynamique ou l' $\alpha$-caténine alternerait continuellement entre une forme monomérique pouvant se lier à la $\beta$-caténine et une dimérique se liant à l'actine. ment au cytosquelette d'actine (voir aussi [32-34]). Les liaisons entre cadhérines sont dites homophiliques car les cadhérines d'une même sous-famille se lient préférentiellement ensemble (Figure 1). Les tissus embryonnaires expriment souvent un groupe spécifique caractéristique suffisait à expliquer l'affinité tissulaire postulée par J. Holtfreter $[10,11]$. Cependant, des tests in vitro ont montré que la force des liaisons homophiliques était peu différente de celle des liaisons hétérophiliques et certaines cadhérines peuvent en fait fonctionner comme des molécules d'adhérence hétérophiliques [12, 13]. La capacité des cellules à ségréger ne peut donc pas s'expliquer simplement par la spécificité de liaison et d'adhérence, et doit être déterminée également par d'autres mécanismes. La spécificité des liaisons homophiliques des cadhérines ne permet pas non plus de comprendre les bases physiques qui gouvernent l'organisation des cellules en différents tissus au cours du développement.

\section{Bases physiques de la morphogenèse :} hypothèse d'adhérence différentielle Dans les années 1960, Malcom Steinberg propose l'hypothèse d'adhérence différentielle [14] : selon cette hypothèse, les tissus embryonnaires sont assimilés à des liquides et les lois physiques qui gouvernent le comportement de deux liquides, l'eau et l'huile par exemple, dirigeraient aussi les réarrangements tissulaires. Un des principes fondamentaux de la thermodynamique prédit qu'un système tend toujours vers l'état le plus stable, ce qui se mesure par une décroissance de son énergie libre. Dans le cas de deux liquides, une partie de l'énergie libre provient des forces de tension qui s'exercent aux surfaces de contact. Cette énergie libre est d'autant plus grande que les forces de tension de surface sont importantes et la surface de contact étendue. Si la tension de surface est importante, l'énergie libre peut être minimisée en réduisant la surface de contact. Dans l'exemple de l'émulsion eauhuile, l'huile, dont la tension de surface est plus importante que celle de l'eau, forme de petites gouttes sphériques (plus petit rapport surface/volume) entourées d'eau. 
Pour démontrer que ces lois physiques s'appliquent aux tissus, l'équipe de M. Steinberg a reproduit les expériences de J. Holtfreter en mesurant la tension de surface de chaque tissu au sein des différents agrégats cellulaires à l'aide d'un tensiomètre

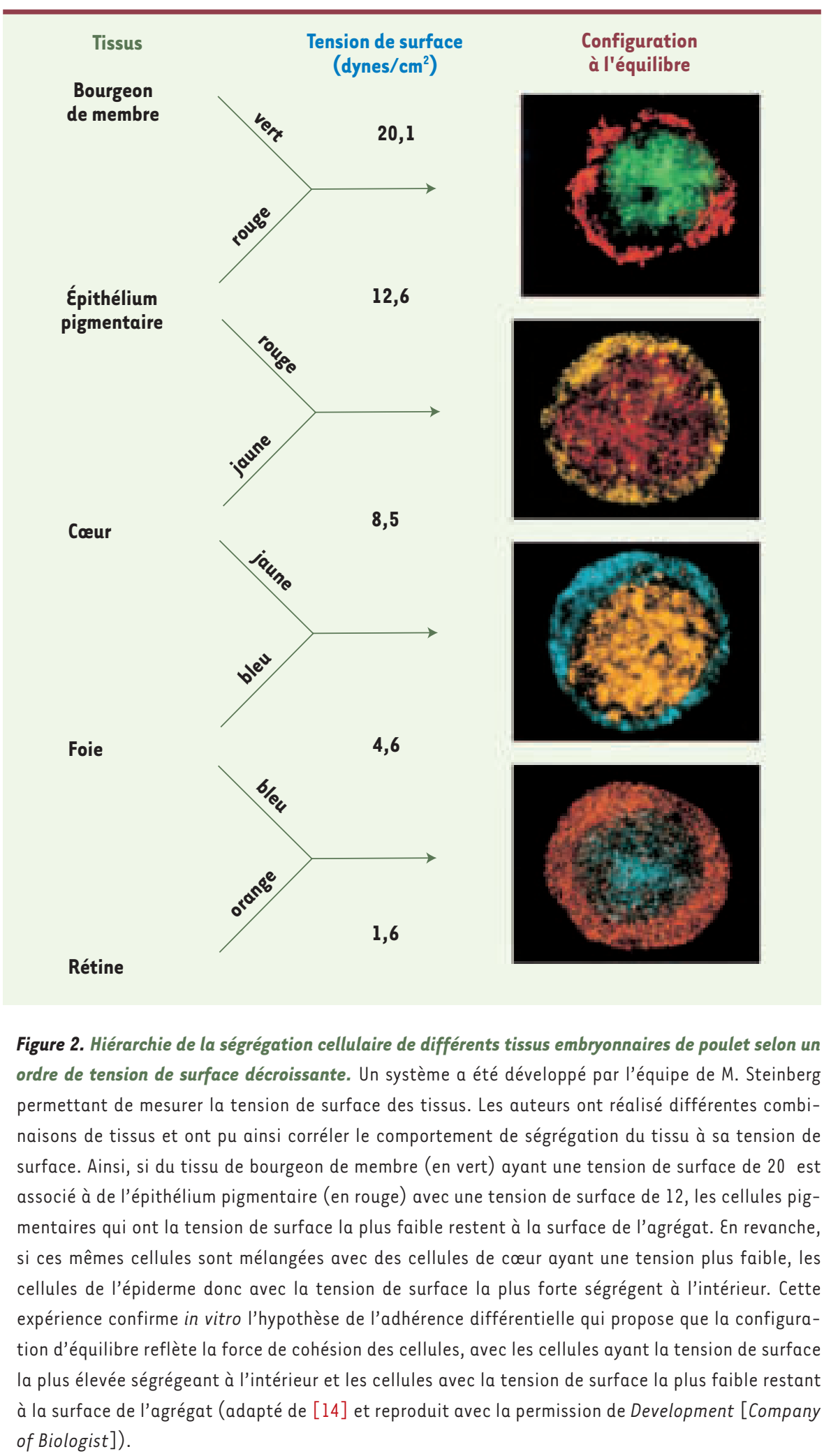

ad hoc (Figure 2). En 1996, il publie des résultats en accord avec l'hypothèse d'adhérence différentielle : quelque soit la combinaison de tissus, le tissu ayant la plus forte tension de surface se trouve toujours au centre de l'agrégat, occupant la surface la plus petite, entouré par le tissu ayant la tension de surface la plus faible et la surface la plus étendue [15]. L'organisation et les mouvements des différents feuillets embryonnaires correspondent donc à l'acquisition de l'état thermodynamique le plus stable. Plus récemment, en 2005, M. Steinberg montre dans un système de culture cellulaire in vitro que la tension de surface tissulaire est directement proportionnelle à la quantité de cadhérines exprimées par les cellules [16]. Ces résultats confèrent une réalité biologique, l'adhérence, à la notion physique de tension de surface, et valident l'hypothèse d'adhérence différentielle. Ce même système in vitro permettra à ces auteurs de montrer que des cellules qui expriment la même cadhérine, mais à des niveaux différents, obéissent aussi à l'hypothèse d'adhérence différentielle, les cellules qui expriment le plus grand nombre de molécules de cadhérine se localisant au centre, entourées par celles qui en expriment moins [16-18]. Ces derniers résultats suggèrent qu'une différence quantitative d'expression d'une même cadhérine suffit pour expliquer «l'affinité tissulaire» différentielle qui dirige l'organisation des tissus, et représente une alternative à la spécificité des liaisons entre cadhérines. Mais si l'hypothèse d'adhérence différentielle permet de rendre compte du comportement de cellules cultivées in vitro au sein d'agrégats artificiels, qu'en est-il pour les mouvements morphogénétiques survenant in vivo au cours du développement d'organismes complexes?

\section{Adhérence différentielle et morphogenèse chez la drosophile}

La drosophile présente de nombreux avantages pour tester l'hypothèse d'adhérence différentielle in vivo: la séquence complète de son génome permet de connaître le nombre et la nature de toutes les cadhérines; des lignées mutantes existent pour la plupart des gènes ainsi identifiés et montrent qu'il y a peu de redondance fonctionnelle; des outils génétiques puissants permettent d'éliminer ou de surexprimer une cadhérine spécifiquement dans un tissu ou même dans une seule cellule. Plusieurs articles récents ont ainsi tiré parti de ces avantages 
pour étudier les phénomènes d'adhérence nécessaires à la détermination de l'axe antéro-postérieur ainsi qu'à la morphogenèse de l'œil.

\section{Gradient d'adhérence}

\section{et mise en place de l'axe antéro-posterieur}

Chez la drosophile, la mise en place des axes de symétrie débute très précocement par la polarisation de l'œuf au cours de l'ovogenèse [19, 20]. Chaque œuf, ou ovocyte, se développe au sein d'une chambre ovarienne composée de 16 cellules germinales, 15 cellules nourricières et un ovocyte, entourées par un épithélium de cellules folliculaires d'origine somatique (Figure 3). Dans cette chambre, l'ovocyte se localise toujours au contact des cellules folliculaires les plus postérieures, avec lesquelles il échange des signaux nécessaires à sa polarisation antéro-postérieure. Cette localisation de l'ovocyte est cruciale, car seules les cellules folliculaires postérieures sont compétentes pour recevoir ces signaux [21]. Le positionnement de l'ovocyte est donc la première étape de la mise en place des axes de polarité chez la drosophile.

En 1998, deux équipes ont exploré le rôle de la $\varepsilon$-cadhérine (epithelial cadherin) dans ce processus morphogénétique [22, 23]. Une observation initiale importante était que l'ovocyte exprime plus de $\varepsilon$-cadhérine que les cellules nourricières et que les cellules folliculaires postérieures expriment également plus de $\varepsilon$-cadhérine que les autres cellules somatiques (Figure 3). Ces observations suggéraient qu'un «tri » cellulaire fondé sur la quantité de $\varepsilon$-cadhérine pourrait être impliqué dans le mouvement de l'ovocyte vers le pôle postérieur de la chambre. Pour le vérifier, les auteurs ont éliminé l'expression de la $\varepsilon$-cadhérine dans la lignée germinale, ce qui entraînait une localisation aléatoire de l'ovocyte dans la chambre ovarienne. Ce même phénotype est aussi obtenu dans les mutants dont toutes les cellules folliculaires sont dépourvues de $\varepsilon$-cadhérine. L'expression de $\varepsilon$-cadhérine dans la lignée germinale et somatique est donc nécessaire au positionnement de l'ovocyte. De manière intéressante, lorsque la $\varepsilon$-cadhérine est éliminée d'une partie seulement des cellules folliculaires, l'ovocyte se positionne toujours au contact des cellules sauvages exprimant la cadhérine, et de préférence vers les cellules les plus postérieures (Figure 3 ). Cela suggère que l'ovocyte est sensible à un gradient d'adhérence présent dans l'épithélium folliculaire [22]. Nous avons récemment testé cette hypothèse en surexprimant directement la $\varepsilon$-cadhérine dans les cellules latérales de l'épithélium folliculaire. L'ovocyte se localise alors toujours au contact des cellules surexprimant la cadhérine, même au sein de cellules folliculaires exprimant la $\varepsilon$-cadhérine endogène (Figure 3) [24]. Ces données montrent que l'ovocyte est capable de distinguer différents niveaux d'expression de $\varepsilon$-cadhérine et que son positionnement dépend de la reconnaissance d'une différence quantitative d'expression d'une même molécule d'adhérence. II s'agit de la première validation in vivo de l'hypothèse d'adhérence différentielle.

\section{A Follicule ovarien sauvage}

Cellules

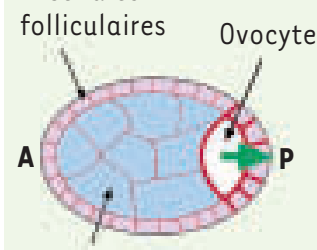

Cellules

nourricières

\section{B Follicule ovarien avec des cellules sans $\varepsilon$-cadhérine}

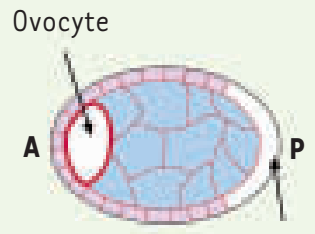

Cellules sans $\varepsilon$-cadhérine
C Follicule ovarien avec des cellules surexprimant la $\varepsilon$-cadhérine

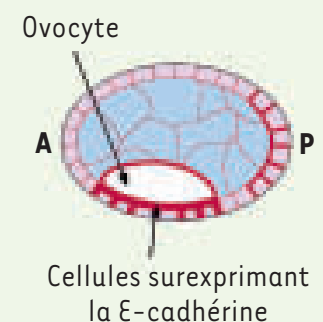

Figure 3. La localisation de l'ovocyte dépend d'un gradient de $\varepsilon$-cadhérine. $A$. Le follicule ovarien est formé de seize cellules d'origine germinale interconnectées, l'ovocyte et les quinze cellules nourricières, entourées par une monocouche de cellules folliculaires, d'origine somatique, et constituant un épithélium continu. Ces deux populations cellulaires ont une organisation stéréotypée où l'ovocyte est toujours localisé au contact des cellules folliculaires postérieures. Cette localisation est cruciale car seules ces cellules sont compétentes pour recevoir les signaux émis par l'ovocyte (flèche verte). Les cellules folliculaires postérieures ainsi que l'ovocyte expriment un niveau plus élevé de $\varepsilon$-cadhérine (en violet). B. La perte de la $\varepsilon$-cadhérine dans les cellules folliculaires postérieures provoque une délocalisation de l'ovocyte qui se retrouve en position antérieure dans cet exemple. L'ovocyte adhère aux cellules qui continuent à exprimer la $\varepsilon$-cadhérine (en violet). C. La surexpression de la $\varepsilon$-cadhérine dans un groupe de cellules folliculaires attire l'ovocyte qui va se localiser au contact de ces cellules exprimant le niveau le plus élevé de cette molécule d'adhérence. Ces données montrent que l'ovocyte est capable de distinguer différents niveaux de $\varepsilon$-cadhérine. La localisation de l'ovocyte dépend donc d'une différence quantitative d'une même molécule d'adhérence.

Comment s'établit une telle différence quantitative de $\varepsilon$-cadhérine? Nous avons montré que la taline, qui normalement se lie aux intégrines, contrôle au niveau transcriptionnel l'expression de la $\varepsilon$-cadhérine $[24,25]$. Mais un second contrôle existe au niveau post-transcriptionnel, puisqu'une expression ubiquitaire de la $\varepsilon$-cadhérine est également suffisante pour créer un gradient d'adhérence au sein de l'épithélium folliculaire [26]. Ces expériences établissent qu'un contrôle génétique de la $\varepsilon$-cadhérine crée des différences quantitatives d'adhérence, elles-mêmes suffisantes pour guider des mouvements morphogénétiques. Mais ce ne sont que des premiers éléments de réponse, et les mécanismes sous-jacents restent encore largement inconnus.

\section{L'œil de drosophile et les bulles de savon}

L'œil de drosophile est un œil composé, formé d'environ 800 yeux simples appelés ommatidies, elles-mêmes comprenant une vingtaine de cellules. L'agencement quasicristallin des ommatidies au sein de l'œil et celui des cellules au sein de chaque omma- 
tidie fait de l'œil de drosophile un formidable modèle pour étudier comment une cellule acquiert une forme spécifique au sein d'un organe complexe. En 2004, T. Hayashi et R.W.Carthew se sont particulièrement intéressés à l'organisation des quatre cellules en cônes (CC) situées au-dessus des cellules photoréceptrices $(P)$ et entourées par des cellules pigmentaires (CP), qui forment la lentille de chaque ommatidie (Figure 4) [27]. Ils ont tout d'abord remarqué que la disposition en losange des quatre cellules en cône était identique à celles de quatre bulles de savon. Or il est bien connu qu'un ensemble de bulles de savon s'organise selon les lois physiques réduisant au minimum l'énergie libre de surface et que le nombre de configurations thermodynamiquement stables est prédit par les lois de Pla-
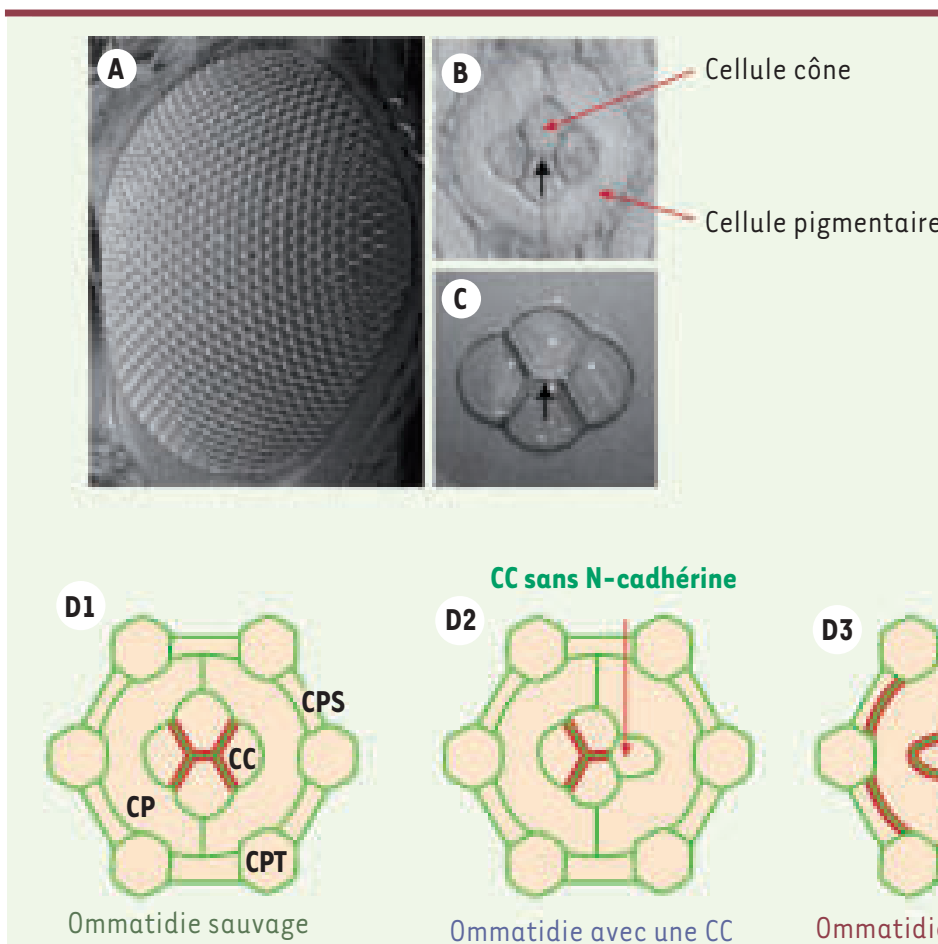

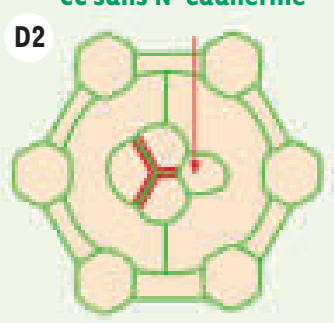

Ommatidie avec une $\mathrm{CC}$ sans $\mathrm{N}$-cadhérine

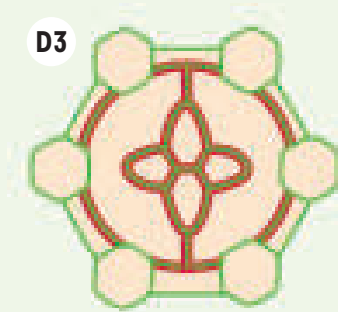

Ommatidie avec deux CP exprimant la $\mathrm{N}$-cadhérine
Figure 4. L'œil de drosophile. A. L'œil de drosophile est un œil composé formé d'environ 800 yeux simples appelés ommatidies. B. Chaque ommatidie est composée d'une vingtaine de cellules. Les cellules cônes (CC) se trouvent au centre, entourées par les cellules pigmentaires (CP). La flèche marque l'interface de jonction entre les cellules cône. Ces CC adoptent une configuration en losange qui rappelle l'organisation de quatre bulles de savon (adapté de [25] et reproduit avec la permission de Nature [McMillan Publishers $\lfloor t d]$ ). $C$. Un groupe de 4 bulles de savon avec une flèche marquant l'interface de jonction entre les bulles (adapté de [25] et reproduit avec la permission de Nature [McMillan Publishers Ltd]). D1. La $\varepsilon$-cadhérine (en vert) est exprimée par toutes les cellules de l'ommatidie et se localise au niveau de toutes les surfaces de contact. La N-cadhérine (en rouge) est exprimée uniquement par les cellules en cône et sa localisation est restreinte aux interfaces entre CC. D2. Si la N-cadhérine est retirée spécifiquement dans une cellule cône (flèche), elle change alors de forme et augmente sa surface de contact avec les CP. D3. L'expression de la $\mathrm{N}$-cadhérine dans les CP entourant les CC entraîne un changement de forme de ce groupe de cellules. Les $C C$ adoptent alors une forme de crucifix plutôt que de losange. Ces résultats démontrent que c'est la différence d'expression de la $\mathrm{N}$-cadhérine entre les CC et les CP qui donne l'organisation spécifique en losange. teau $^{1}[28]$. Afin de tester si les mêmes lois gouvernaient les cellules en cône, les auteurs ont utilisé le mutant rough eye (Roi), caractérisé par un nombre variable et anormal de CC ( 1 à 6 par ommatidie). De manière remarquable, quel que soit le nombre de CC, les cellules adoptent toujours les configurations prédites par la thermodynamique, indiquant qu'une «minimisation » de la tension de surface guide aussi l'organisation quasi-cristalline de l'œil. L'analyse du profil d'expression des cadhérines révèle que la $\varepsilon$-cadhérine est exprimée par toutes les cellules de l'ommatidie et se localise à toutes les surfaces de contact, alors que la $\mathrm{N}$-cadhérine est restreinte aux cellules en cône et ne se localise qu'aux interfaces CC-CC (Figure 4). Ces observations suggèrent que le profil d'expression de la $\mathrm{N}$-cadhérine serait responsable de l'adhérence différentielle entre les cellules en cône et les cellules pigmentaires qui les entourent. De fait, l'élimination spécifique de la N-cadhérine des CC (créant un niveau uniforme de $\varepsilon$-cadhérine entre les $C C$ et les $(P)$ induit les CC à adopter une forme de crucifix plutôt que de losange. La même forme en crucifix est obtenue lorsque l'expression ectopique de la $\mathrm{N}$-cadhérine est induite dans les CP (créant un niveau uniforme de $\varepsilon-+\mathrm{N}$ cadhérine entre $C($ et $(P)$. C'est donc bien la différence d'expression de la $\mathrm{N}$-cadhérine entre les CC et les CP qui confère une forme spécifique à la lentille (Figure 4) [27]. Par ailleurs, une surexpression de la $\varepsilon$-cadhérine dans les CP (créant un niveau uniforme de «cadhérine» entre $(C$ et $(P)$ n'induit pas de changement de forme. L'adhérence différentielle entre CC et CP n'est donc pas d'origine quantitative mais qualitative. Les auteurs en concluent que la forme spécifique des cellules en cône est à la fois sous contrôle génétique et physique. La régulation génétique permet d'exprimer des cadhérines qualitativement différentes dans des cellules spécifiques, alors que les lois physiques minimisent l'énergie de surface pour donner à la lentille sa forme finale.

\section{Conclusions et perspectives}

L'idée que des principes physiques simples peuvent gouverner la formation de structures biologiques n'est pas nouvelle [29]. Cependant, sa démonstration s'est souvent heurtée à l'extraordinaire variété et complexité

${ }^{1}$ NDLR : En 1873, J. Plateau publia un traité sur les films minces, dans lequel il énonçait des règles expérimentales gouvernant l'agencement de films de savon dans différentes structures. Elles sont une conséquence directe du principe de «minimisation» de l'énergie. Ces règles sont connues sous le nom de règles de Plateau, et s'énoncent ainsi : (1) Trois films de savon se rejoignent à angle de $120^{\circ}$, pour former un bord appelé bordure de Plateau. (2) En un point, se rejoignent au plus quatre bords, formant entre eux l'angle d'un tétraèdre régulier (simulation géométrique et optique des mousses savonneuses. Didier Arquès, Isabelle Icart). 
des formes du vivant. Les deux exemples que nous avons abordés montrent qu'une régulation génétique simple de l'expression des cadhérines au niveau quantitatif et qualitatif, ainsi qu'un principe physique élémentaire, celui de la «minimisation» de l'énergie de surface, permettent de rendre compte de processus aussi complexes que la mise en place de l'axe antéro-postérieur ou la morphogenèse de l'œil chez la drosophile. La diversité des formes ne saurait cependant se réduire uniquement à des différences d'expression de molécules d'adhérence. Le cytosquelette joue également un rôle fondamental, car il permet à la cellule non seulement d'acquérir une forme véritablement stable, mais aussi de changer de forme, en remaniant les jonctions adhérentes au cours des mouvements morphogénétiques [30]. Cette régulation dynamique de l'adhérence est cruciale lorsque les cellules changent de voisines, par exemple, lors de l'élongation de l'embryon de drosophile ou de manière plus générale lors de la gastrulation [31]. L'étude de la morphogenèse se poursuit donc au niveau des mécanismes cellulaires, qui eux intègrent les régulations génétiques et physiques. $\diamond$

\section{SUMMARY}

Genetic control of intercellular adhesion

or how cadherins shape the fruitfly Drosophila melanogaster

The beauty and diversity of cell shapes have always fascinated both biologists and physicists. In the early 1950, J. Holtfreter coined the term «tissue affinities » to describe the forces behind the spontaneous shaping of groups of cells. These tissue affinites were later on related to adhesive properties of cell membranes. In the 1960, Malcom Steinberg proposed the differential adhesion hypothesis (DAH) as a physical explanation of the liquid-like behaviour of tissues and cells during morphogenesis. However, the link between the cellular properties of adhesion molecules, such as the cadherins, and the physical rules that shape the body, has remained unclear. Recent in vitro studies have now shown that surface tensions, which drive the spontaneous liquid-like behaviour of cell rearrangements, are a direct and linear function of cadherin expression levels. Tissue surface tensions thus arise from differences in intercellular adhesiveness, which validates the DAH in vitro. The DAH was also vindicated in vivo by stunning experiments in Drosophila. The powerful genetic tools available in Drosophila allow to manipulate the levels and patterns of expression of several cadherins and to create artificially differences in intercellular adhesiveness. The results showed that simple laws of thermodynamics, as well as quantitative and qualitative differences in cadherins expression were sufficient to explain processes as complex as the establishment of the anterior-posterior axis and the formation of the compound eye in Drosophila. $\diamond$

\section{REMERCIEMENTS}

Nous remercions le Dr Malcom Steinberg pour la Figure 2 et le Dr Jean-Yves Roignant pour la photo d'œil de drosophile de la Figure 4.

\section{RéFÉRENCES}

1. Wilson HV. One some phenomena of coalescence and regeneration in sponges. J Exp Zool 1907 ; $5: 53-120$.

2. Townes PL, Holtfreter J. Directed movements and selective adhesion of embryonic amphibians cells. J Exp Zool 1955; $128: 53-120$.

3. Steinberg MS. Adhesion in development: an historical overview. Dev Biol 1996; $180: 377-88$.

4. Brackenbury R, Thiery JP, Rutishauser U, et al. Adhesion among neural cells of the chick embryo. I. An immunological assay for molecules involved in cell-cell binding. J Biol Chem $1977 ; 252: 6835-40$.
5. Thiery JP, Brackenbury R, Rutishauser U, et al. Adhesion among neural cells of the chick embryo. II. Purification and characterization of a cell adhesion molecule from neural retina. J Biol Chem 1977 ; 252 : 6841-5.

6. Hyafil F, Babinet C, Jacob F. Cell-cell interactions in early embryogenesis: a molecular approach to the role of calcium. Cell 1981;26:447-54.

7. Bokel C, Brown NH. Integrins in development: moving on, responding to, and sticking to the extracellular matrix. Dev Cell $2002 ; 3: 311-21$.

8. Tepass U. Genetic analysis of cadherin function in animal morphogenesis. Curr Opin Cell Biol 1999; $11: 540-8$.

9. Wheelock MJ, Johnson KR. Cadherins as modulators of cellular phenotype. Annu Rev Cell Dev Biol 2003; $19: 207-35$.

10. Nose A, Nagafuchi A, Takeichi M. Expressed recombinant cadherins mediate cell sorting in model systems. Cell 1988; $54: 993-1001$.

11. Takeichi M. Cadherin cell adhesion receptors as a morphogenetic regulator. Science $1991 ; 251: 1451-5$.

12. Duguay D, Foty RA, Steinberg MS. Cadherin-mediated cell adhesion and tissue segregation: qualitative and quantitative determinants. Dev Biol 2003 ; $253: 309-23$.

13. Niessen CM, Gumbiner BM. Cadherin-mediated cell sorting not determined by binding or adhesion specificity. J Cell Biol 2002 ; 156 : 389-99.

14. Steinberg MS. Reconstruction of tissues by dissociated cells. Some morphogenetic tissue movements and the sorting out of embryonic cells may have a common explanation. Science 1963 ; 141: 401-8.

15. Foty RA, Pfleger CM, Forgacs G, et al. Surface tensions of embryonic tissues predict their mutual envelopment behavior. Development 1996; 122 : 1611-20.

16. Foty RA, Steinberg MS. The differential adhesion hypothesis: a direct evaluation. Dev Biol $2005 ; 278: 255-63$.

17. Steinberg MS, Takeichi M. Experimental specification of cell sorting, tissue spreading, and specific spatial patterning by quantitative differences in cadherin expression. Proc Natl Acad Sci USA 1994 ; 91 : 206-9.

18. Friedlander DR, Mège RM, Cunningham BA, et al. Cell sorting-out is modulated by both the surface specificity and amount of different cell adhesion molecules (CAMs) expressed on cell surfaces. Proc Natl Acad Sci USA 1989; 86 : 7043-7.

19. Huynh JR, St Johnston D. The origin of asymmetry: early polarisation of the Drosophila germline cyst and oocyte. Curr Biol 2004; 14 : R438-49.

20. Riechmann V, Ephrussi A. Axis formation during Drosophila oogenesis. Curr Opin Genet Dev $2001 ; 11$ : 374-83.

21. Gonzalez-Reyes A, St Johnston D. Patterning of the follicle cell epithelium along the anterior-posterior axis during Drosophila oogenesis. Development $1998 ; 125: 2837-46$.

22. Godt D, Tepass U. Drosophila oocyte localization is mediated by differential cadherin-based adhesion. Nature $1998 ; 395: 387-91$.

23. Gonzalez-Reyes A, St Johnston D. The Drosophila AP axis is polarised by the cadherin-mediated positioning of the oocyte. Development 1998 ; 125 : 3635-44.

24. Becam IE, Tanentzapf G, Lepesant JA, et al. Integrin-independent repression of cadherin transcription by talin during axis formation in Drosophila. Nat Cell Biol $2005 ; 7: 510-6$.

25. Nayal A, Webb DJ, Horwitz AF. Talin: an emerging focal point of adhesion dynamics. Curr Opin Cell Biol $2004 ; 16: 94-8$.

26. Pacquelet $A$, Rorth $P$. Regulatory mechanisms required for $D E$-cadherin function in cell migration and other types of adhesion. J Cell Biol 2005; $170: 803-12$.

27. Hayashi T, Carthew RW. Surface mechanics mediate pattern formation in the developing retina. Nature $2004 ; 431: 647-52$.

28. Plateau JAF. Statique experimentale et théorique des liquides soumis aux seules forces moléculaires. Paris: Gauthier-Villars, 1873.

29. D’Arcy Thompson R. On growth and form. Cambridge : Cambridge University Press, 1969.

30. Lecuit T. Adhesion remodeling underlying tissue morphogenesis. Trends Cell Biol 2005 ; $15: 34-42$.

31. Bertet C, Sulak L, Lecuit T. Myosin-dependent junction remodelling controls planar cell intercalation and axis elongation. Nature 2004 ; 429 : 667-71.

32. Drees F, Pokutta $S$, Yamada S, et al. Alpha-catenin is a molecular switch that binds $\varepsilon$-cadherin-beta-catenin and regulates actin-filament assembly. Cell $2005 ; 123: 903-15$.

33. Gates J, Peifer M. Can 1000 reviews be wrong? Actin, alpha-catenin, and adherens junctions. Cell 2005; 123: 769-72.

34. Yamada S, Pokutta S, Drees F, et al. Deconstructing the cadherin-cateninactin complex. Cell $2005 ; 123: 889-901$.

\section{TIRÉS À PART}

J.R. Huynh 